\title{
Virtual benchmarking in logistics: A concept for the determination of benchmarks
}

\author{
Volker Hornung, Holger Luczak, Marcel Oster \\ Research Institute for Operations Management (FIR) \\ Aachen University of Technology \\ Pontdriesch 14/16 \\ 52062 Aachen, Germany \\ Tel: +49-241-47705-62, Fax: +49-241-402401 \\ os@fir.rwth-aachen.de
}

\begin{abstract}
Benchmarking has been discussed controversially in the past as a method to learn about processes of other companies known for their outstanding capabilities. Identifying best-in-class companies in specific processes is difficult. Comparable benchmarks are scarce and influential factors have a large impact on the performance. Therefore the virtual benchmarking concept has been developed. First step was to define the objectives in logistics. In order to transfer the objectives into processes, a set of top priority logistics measures along with influential factors has been developed and validated. The concept was filled with empirical data from more than 120 companies and transformed into a software tool called LOGCHECK. It presents an appropriate possibility to gain information about benchmarks in logistics, depending on the conditions influencing a company. The virtual benchmarks are immediately available after an analysis of the respective company's conditions. The database is constantly updated.
\end{abstract}

\section{Keywords}

Benchmarking, Logistics, Measures, Influential Factors, Virtual Benchmarking

Advances in Production Management Systems N. Okino, H. Tamura \& S. Fujii (Eds.)

(C) 1998 IFIP. Published by Chapman \& Hall 
With more complex production processes, increasing automation, numbers of products, and variety leaving limited possibilities to improve production, companies are facing and focusing on higher demands of their logistic performance. The perceptible performance of logistics processes such as delivery service, delivery reliability or fast response determines today, beside price and product quality, the difference to competitors. The quality of logistics performance and services becomes more important as design and product features become more and more similar and comparable. But leadership in logistics performance should not be bought by high costs in logistics for large stocks etc. What must therefore be found are efficient, innovative processes meeting highest customer expectations at low costs, known as to be reliable. The questions to be answered are: What process quality is achievable and what are the processes leading to superior performance? Benchmarking tries to answer those questions, allowing and promoting new ideas while setting realistic targets.

\subsection{Benchmarking Process}

The goal of benchmarking is to compare products, methods or processes of different companies or divisions, to evaluate the weak points and to find the best processes and methods applicable in the own company: best practices. A benchmarking study should be made in co-operation with companies known as best-in-class concerning the subject to be compared. Benchmarking is also used to learn how that excellence is achieved by the best-in-class companies. Therefore, the collection of quantitative data must be followed by the identification of reasons for a superior performance. Final goal is not only to obtain measures and identify the gap, but to even surpass the measured performance and become the best by adapting the best practices considering the specific conditions of the own company.

The partners should be the best-in-class and must operate under comparable conditions. Finding the best-in-class can be a difficult task, persuading them to participate in a benchmarking study even more. Companies have difficulties revealing how they work to others.

The selection of the right benchmarking partner plays a key role for the success of a benchmarking study. Two basic principles can be distinguished:

- Internal benchmarking with own separate divisions, profit centers, plants or departments and

- external benchmarking with different companies acting in the same or different industries.

Each of them has advantages, and especially the possibility of getting reliable data from other subsidiaries should not be neglected. The price of being able to 
gather data very quickly with internal benchmarking is the limited angle of view when only different subsidiaries are compared with one another.

\subsection{Benchmarking and Logistics}

The quality of logistics processes strongly depends on the quality and the availability of information offering possibilites to learn about the present state. This often leads to questions as:

- What are the potentials of our logistics?

- Is our stock level too high compared with others?

- Are our logistics costs accurate?

- How can those information be quantified?

Success factors have extended from the two dimensions quality and costs to the third dimension logistics performance. Taking into account that the share of logistic costs in turnover can reach up to $30 \%$ underlines why an optimized logistic performance by reduced costs is of major interest in global markets. Benchmarking in logistics can help answering these questions.

\subsection{Classical Data Sources for Benchmarks}

How can measures about the performance of others and especially information about best-in-class be obtained? The three basic principles are shown in Figure 1.

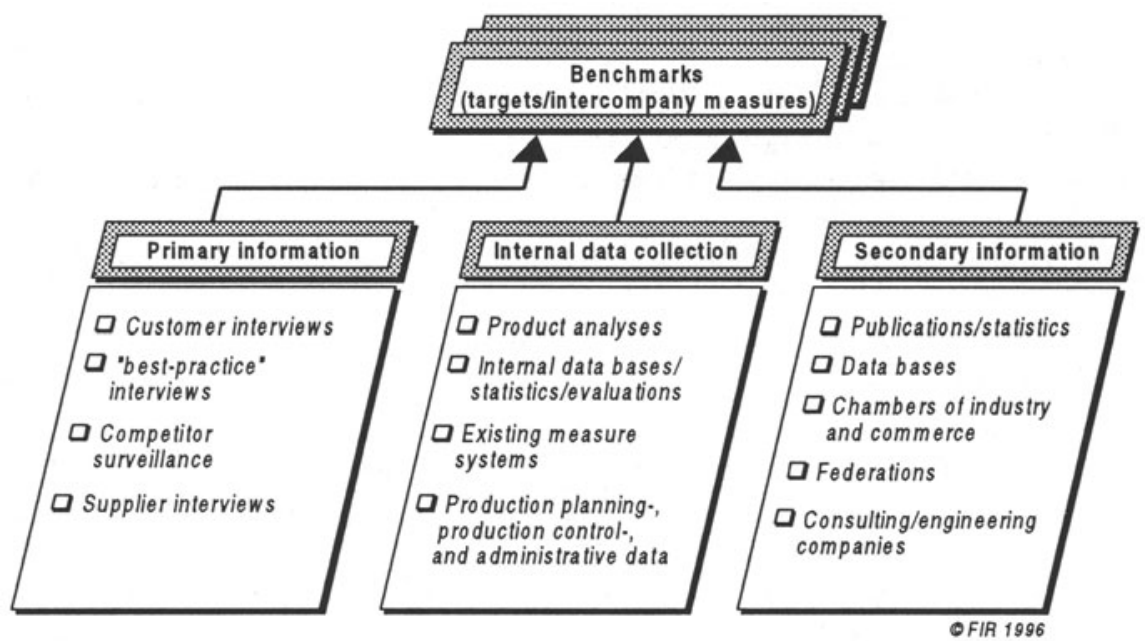

Figure 1 Data sources 


\subsection{Problems with the Realization}

The realization of positive effects is as difficult as the benchmarking principle sounds promising.

- Benchmarking is based on mutuality. Since a benchmarking study usually is not done anonymously between two companies, the selection of the partner and the collection of the partly very sensible data causes a lot of problems. Only the comparison with a better or the best is useful, but companies must be persuaded to participate. All the involved parties must draw their advantages from the study. This is the reason why external studies are normally only conducted between companies not being direct competitors in the same market.

- If a company decides to carry out an external benchmarking study, it often lacks the knowledge of who is the best-in-class in other markets because differences in the subjects to compare may have an impact on the comparison.

- Not to be neglected is the investment of time and workforce necessary to accomplish a successful benchmarking study.

Those are some reasons, why in Europe and especially in Germany benchmarking is not widely used and if so, it is often only done between subsidiaries of large scale companies. Most of them are subsidiaries from American companies.

A recent study and contacts with company representatives revealed that companies although they are of large scale, well known and/or multinational oriented still have problems finding benchmarking partners. If they find a partner, they prefer to keep their experience of how to conduct a benchmarking study secret. This explains the motivation for the work undertaken. There is a need for the immediate, cost effective determination of benchmarks from really comparable companies.

\section{VIRTUAL BENCHMARKING CONCEPT}

Since partners are hard to find and the benchmarking process is not widely accepted, the „virtual“ benchmarking concept has been developed at the Forschungsinstitut für Rationalisierung (FIR) at Aachen University of Technology (RWTH Aachen) using a different approach. The concept tries to avoid the above mentioned problems. Starting point has been a research project made to determine the influential factors on measures in logistics, independent from the industrial sector. Sector measures are usually distributed by federations or statistical offices. Such measures only have a limited benefit for practical use when comparing different companies since the influencing factors vary from company to company as well as the measure definitions. Furthermore they normally present average values and do not offer any data for best practice performance. 
Usually, only isolated measures (having few connections between each other) are taken but rarely embedded in a measurement system. Another main problem is that only mean values for whole industrial sectors are available, not reflecting the influential factors representing the real situation a company is in. Influential factors such as requirements explosion, production program planning support, structure of suppliers and customers etc. are not considered. Therefore, the comparison of measures between two or more companies is limited to average values.

\subsection{Empirical Basis}

The objectives of the research project were the derivation of a measurement system for intercompany comparison in logistics followed by an evaluation of the factors having an influence on the measures and the development of intercompany measures. Figure 2 shows the steps of the research project that lead to the software tool LOGCHECK.

Starting point was the development of an objective's system for logistics. Based on the main objectives of a company, the objectives in logistics were derived. They can be separated into performance objectives and cost objectives. Performance objectives are: to ensure the market supply, to ensure production supply, and to improve amount and delivery reliability. Cost objectives are: optimized stocks and work in progress, reduced lead times, optimized capacity utilization and reduced logistics costs. Those objectives are independent from a specific company or an industrial sectors. In order to maintain this general validity the objectives were not subdivided further.

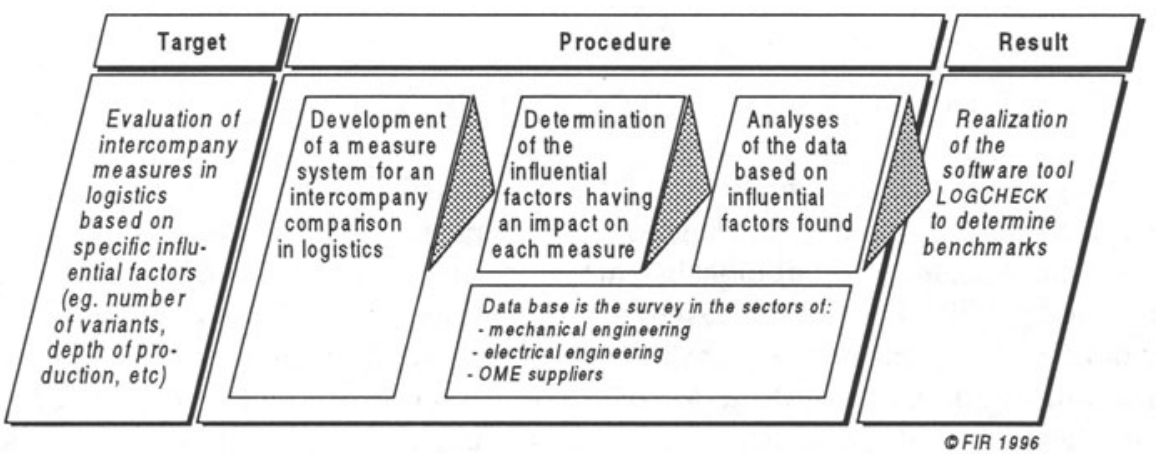

Figure 2 Project steps

The next step was to develop a measure system. A first study lead to a catalogue of more than 200 logistics measures. They were checked for general validity, relevance in practice and possibilities to record them as well as the degree of their coverage of logistics. Based on discussions with experts and a 
comparison with the objectives a set of 23 measures was derived. The measures derived are for example: delivery reliability of finished goods or delivery reliability of spare parts, range of finished goods, range of raw material and production supplies, rate of delay or incorrect supplies towards the customer, rate of delivery delay, utilization rates of production means, share of stock in turnover or share of logistics costs in turnover etc. Especially the share of logistics costs in turnover created difficulties during the analysis due to different types of cost and varying cost centers from company to company. Therefore the incomparable parts as e.g. costs for delivery, which is only depending from the distance between supplier and customer, were excluded for the calculation.

The value of a measure strongly depends on the characteristics of a company and the market conditions under which it is working. An intercompany comparison can therefore only be valid, if similar types of companies are compared one with another. For a comparison significant influential factors must be taken into account. Again a first study revealed a catalogue of more then 200 influential factors which were also checked for general validity, relevance in practice, and clearness. This lead to 35 potential influential factors such as type of order, type of production, type of product structure, type of stockpiling etc. Each influential factor is described through up to four characteristics. Some characteristics combinations are also allowed.

Now that a catalogue of measures and a catalogue of potential influential factors existed, the next step was to determine the influence of the influential factors on each measure. Two questions had to be answered. First which of the potential influential factors does really have a statistical significant influence on measure values, and second how can, based on the results of the first question, intercompany measures be derived?

A data collection was carried out via a questionnaire sent to mainly small and medium sized enterprises of different industrial sectors. The standardized questionnaire includes the 23 measures and the 35 influential factors together with its precise definitions. The database build with the results of the questionnaire now contains more than 120 companies and each time a company participates in a new study its data are added to the database.

Based on these data, a significance analysis was done, using statistical KruskalWallis-tests to find those factors, having a statistical valid influence on measures. For each measure it was tested, whether each potential influential factor has a significant influence. After that, each of the 23 measures was evaluated for every of the significant influential factors. In Figure 3 significant influential facotrs for some measures are shown. The complete table is the basis for calculating the „virtual“ benchmarks. This measure catalogue allows to draw individual measures for each type of company with every possible influential factor combination. For every measure not only the mean and the median value can be calculated, but also a tolerance range. The tolerance range is defined through the $25 \%$ percentile and the $75 \%$ percentile. The result of the calculation is a 
comparison of the current state of a company with the 'should be' state given through the combination of the other companies.

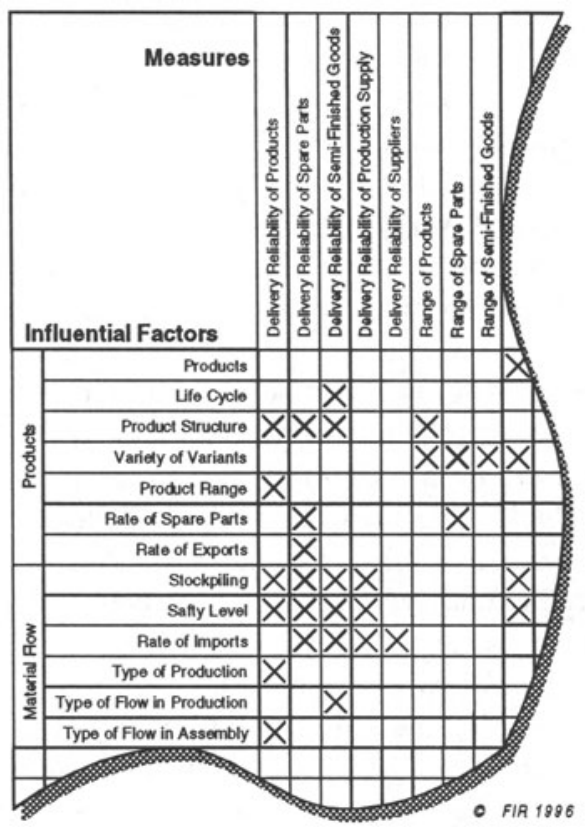

Figure 3 The data base

The measures can be calculated independently from the industrial sector. Like in benchmarking, the independence from the industrial sector is one of the key advantages.

\subsection{Virtual Companies and Virtual Benchmarking}

In a second step the concept was extended to not only calculating average values and tolerance ranges, but also benchmarks by finding out maxima or minima, depending on the measure to be compared.

A comparison between companies can be done in different ways. A direct comparison assumes, that a best-in-class company regarding the process to be improved is found, and this company is willing to participate in a comparison. The anonymous comparison is carried out by a neutral third party (usually consultants) providing data of comparable companies. The comparison with „virtual“ companies uses the survey based data to generate a „virtual“ company with comparable influential factors. The analogy between the current state and how it 'should be' is what leads to the term ,virtual“ companies. In contrast to the 
results of one's own ,real“ company, a „virtual“ company is built through a combination of comparable companies. A company that does not exist in reality. It is built through the results of the survey. The comparison of both benchmarking methods is shown in (Figure 4).

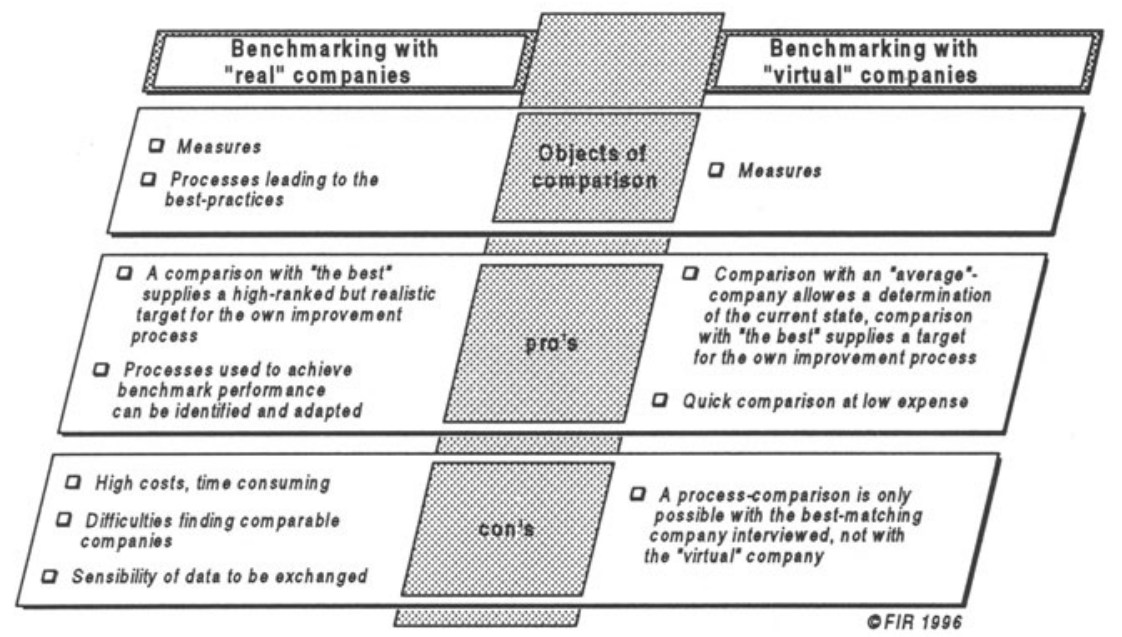

Figure 4 Benchmarking ,real" versus benchmarking „virtual“ companies

The data base does not only contain average „virtual“ companies but also „virtual" best-in-class data: the benchmarks (Figure 5). These benchmarks will also be calculated by taking into account the influential factors: benchmarks from comparable but not existing companies. It is near to impossible to find a real company being able to fit all the influential factors. The virtual best-in-class company is a combination of the best processes of all companies that participated in the survey. The data base is constantly updated with new data.

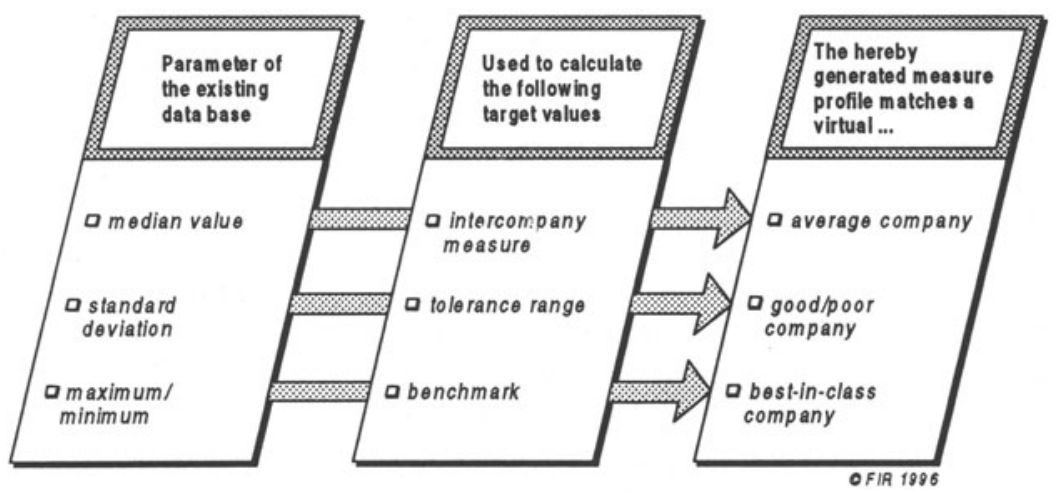

Figure 5 The data base 
Instead of comparing average companies as it was done so often in the past, it is now possible to realize comparisons with best-in-class companies immediately and see which level of excellence they are able to achieve. The virtual best company sets benchmarks. These are realistic and achievable goals for a process optimization. Goals that can be communicated to every employee and will be better accepted than a „we have to improve by $30 \%$ " slogan from the top management.

Virtual benchmarking compares measures and clearly indicates performance gaps. It does not compare processes as the classical benchmarking does.

\subsection{LOGCHECK Concept}

The data base and the calculation logic are used to set up the LOGCHECK concept and to build the software tool LOGCHECK. The LOGCHECK concept is based on four steps, shown in Figure 6.

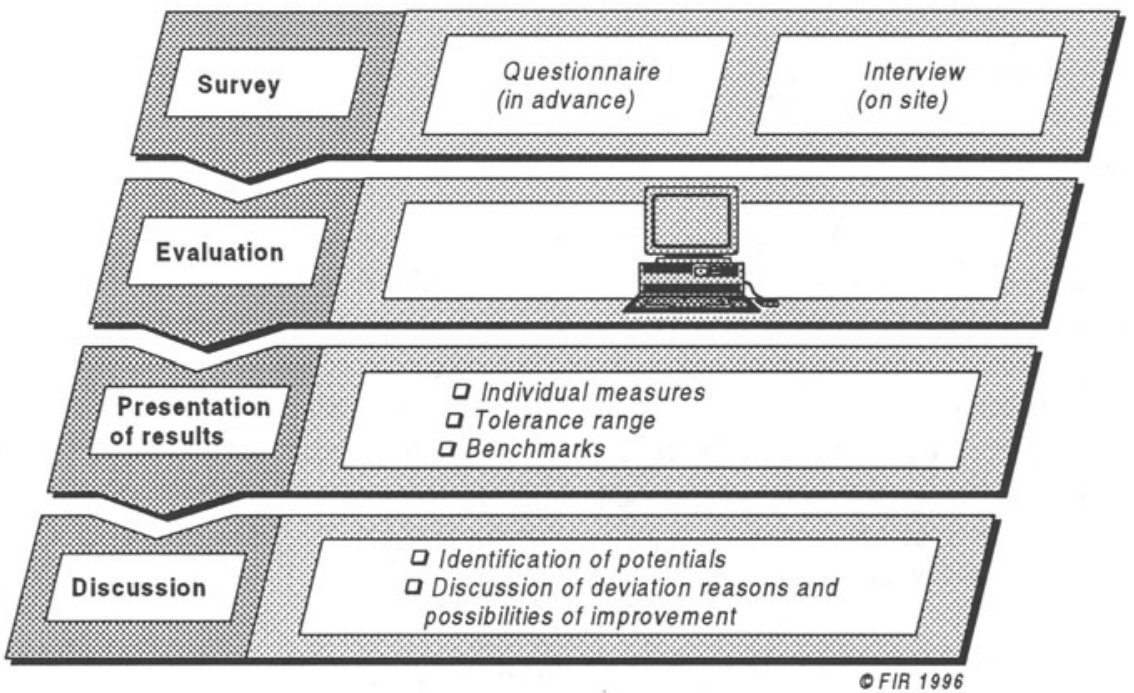

Figure 6 The LOGCHECK procedure

The questionnaire will be sent to the company. First step of the concept is to fill out the questionnaire sent in advance. It does include all the measures as well as it's precise definitions. This is followed by an on-site interview. The on-site interview has proved as to be very important when discussing the results of the study. The data are used to calculate intercompany measures and benchmarks. The starting screen (see Figure 7) shows some of the influential factors considered for the comparison. The influential factors shown are used to determine market position and variety of variants. 


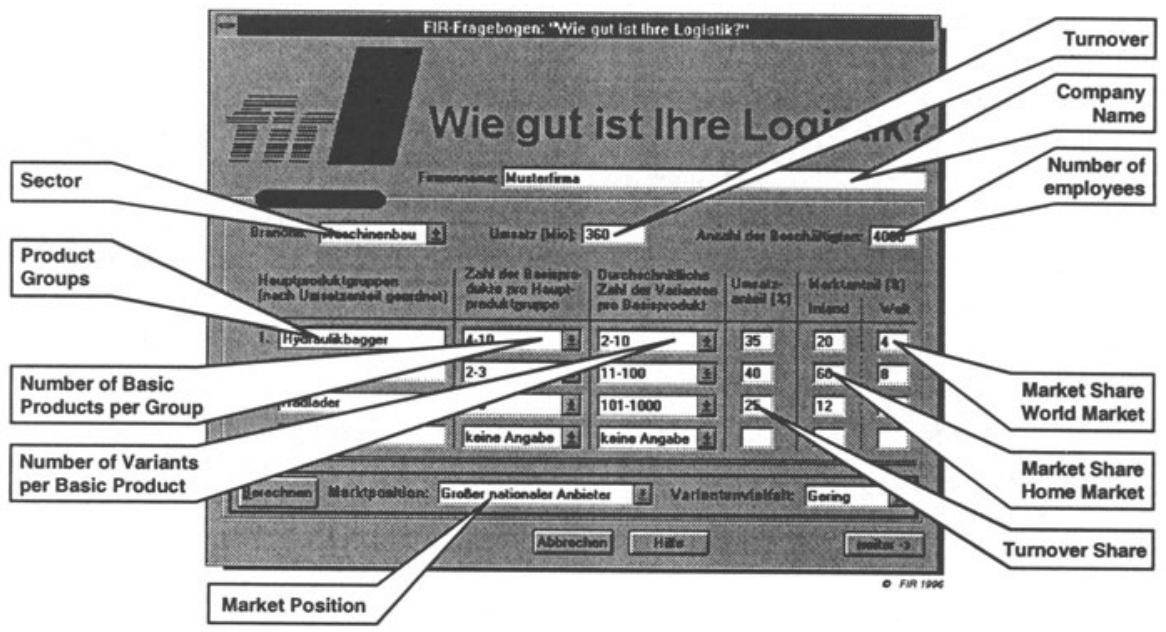

Figure 7 The LOGCHECK software tool

The results are presented to the company and discussed to identify potentials for improvements. Each company having its own influential factor profile uses its specific influential factors to calculate its individual intercompany measures and benchmarks (Figure 8). The management receives information on other companies performance. The expenditure for this comparison is much lower than for a classical benchmarking study.

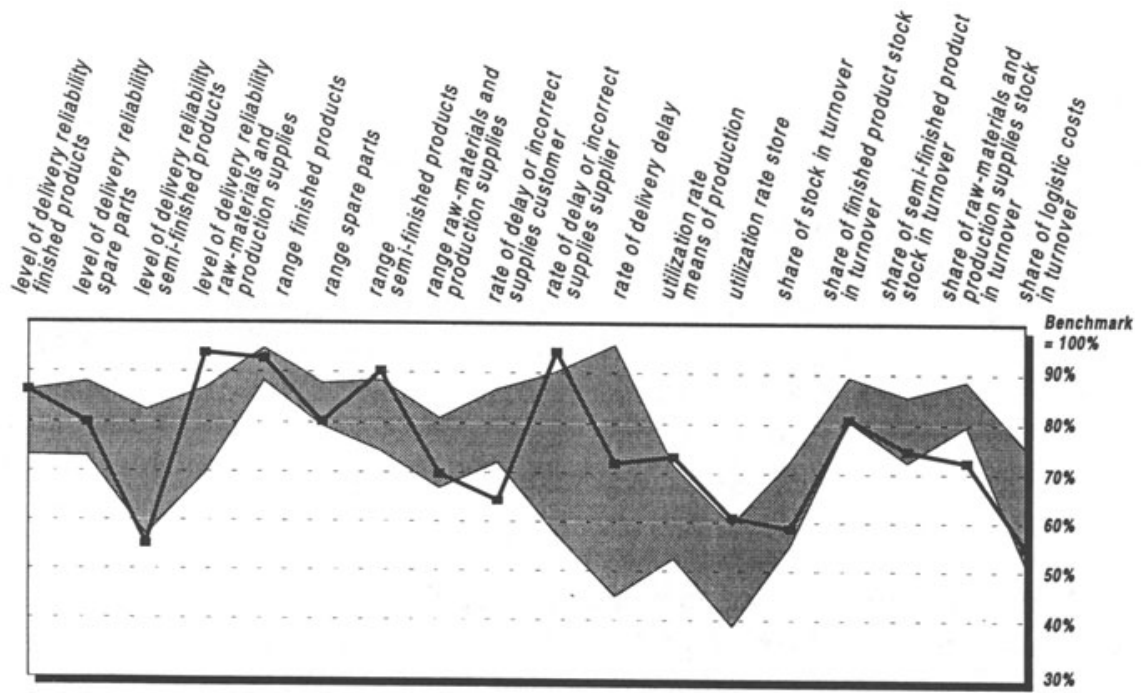

O FIR 1996

Figure 8 Result of LOGCHECK 


\section{CONCLUSION}

The LOGCHECK concept and the underlying model is a sophisticated and efficient instrument to obtain information about the performance of business processes in logistics. The „virtual“ benchmarks are directly available and accessible. The search for partners to learn how well they perform is not necessary and the measures are comparable, since all companies in the database used the same measure definitions. Another positive aspect is that data about the performance of competitors in the same industrial sector can be analyzed although all the data are handled anonymously.

The concept has been successfully applied by various companies. After identifying the performance gaps, they initiated optimization projects in areas where the highest potentials for improvement were located.

\section{REFERENCES}

Abels, H., Anagnostou, E., Brockmann, K.-H. (1994), Wie gut ist Ihre Logistik?, Verlag TÜV Rheinland, Köln.

Pfohl, H.-C. 1985), Logistik-Systeme, betriebswirtschaftliche Grundlagen, Springer Verlag, Berlin et al.

Syska, A. (1990), Kennzahlen für die Logistik, Springer Verlag, Berlin et al.

Watson, G. (1993), Benchmarking, Vom Besten Lernen, Verlag Moderne Industrie, Landsberg/Lech.

\section{BIOGRAPHY}

Dr. Volker Hornung received his Dipl.-Ing. degree in mechanical engineering from Karlsruhe Fridericiana University of Technology, Karlsruhe, Germany in 1981 and the Dipl.-Wirt. Ing. degree in industrial engineering from Aachen University of Technology (RWTH), Aachen, Germany in 1984. After leaving he worked 1984 to 1991 as research engineer and chief engineer in charge of the Institute of Industrial Engineering and Ergonomics (IAW) of the Aachen University of Technology (RWTH) he received his Dr.-Ing. degree in 1991. From 1992 to 1996 he was Managing Director of the Research Institute for Operations Management (FIR), Aachen University of Technology (RWTH).

Prof. Holger Luczak studied mechanical and industrial engineering at Darmstadt University of Technology, Darmstadt, Germany, where he graduated in 1974 to Dr.-Ing. From 1977 to 1983 he was working as full professor at Bremen University, Bremen, Germany. From 1983 to 1992 he was full professor of the Institute for Ergonomics and Industrial Engineering of Berlin, Berlin, Germany. Since 1992 he is working as Director and Chair of the Institute of Industrial 
Engineering and Ergonomics (IAW) of Aachen University of Technology, Aachen, Germany and as Scientific Director of the Research Institute for Operations Management (FIR) at Aachen University of Technology.

Marcel Oster received his Dipl.-Ing. degree in mechanical engineering and the Dipl.-Wirt. Ing. degree in industrial engineering from Aachen University of Technology (RWTH), Aachen, Germany, in 1992 and 1994, respectively. In 1992 he worked as a research trainee at L'Air Liquide, Paris, France. From 1994 to 1995 he was an engineer in the European Pre Order Department with 3M-Germany, Neuss, Germany. Since 1995 he is working as research engineer for the Research Institute for Rationalization and Operations Management (FIR), Aachen University of Technology (RWTH). 